\title{
Mechanism of NHX1 Inhibiting TRPV5-Mediated Podocyte Injury in Diabetic Nephropathy
}

\author{
Rui Li ${ }^{*}$, Qingfen Wang, Xiaochun Niu, Ruirui Xu \\ Department of Nephrology, Binzhou People's Hospital, Binzhou, China
}

Email address:

lirui201812@163.com (Rui Li)

${ }^{*}$ Corresponding author

To cite this article:

Rui Li, Qingfen Wang, Xiaochun Niu, Ruirui Xu. Mechanism of NHX1 Inhibiting TRPV5-Mediated Podocyte Injury in Diabetic Nephropathy. American Journal of Clinical and Experimental Medicine. Vol. 6, No. 5, 2018, pp. 113-117. doi: 10.11648/j.ajcem.20180605.12

Received: November 27, 2018; Accepted: December 5, 2018; Published: December 6, 2018

\begin{abstract}
To observe the effect of high glucose stimulation on the expression of guanylate exchange factor NHX1 in mouse kidney cells, and to explore the role of NHX1 in high glucose-induced podocyte injury and its possible molecular mechanism. The expression of NHX1 in podocytes of diabetic nephropathy patients was observed by immunofluorescence staining and laser confocal microscopy. The immortalized podocytes were cultured in vitro, and the podocytes were stimulated with high glucose for $48 \mathrm{~h}$. RT-PCR, Western blot and immunization were used. Fluorescence detection of mRNA and protein expression of NHX1 in podocytes stimulated by high glucose; Western blot, immunofluorescence and scratch assay were used to detect the expression of NHX1 and the expression of podocin, the activity of podocytes and the nuclear access of TRPV5. The transcription of the target gene downstream of TRPV5 was detected by RT-PCR. NHX1 expression was significantly decreased in podocytes and high glucose-stimulated podocytes of diabetic nephropathy patients $(\mathrm{P}<0.05)$. After silencing NHX1, podocyte marker protein podocin expression was significantly decreased and podocyte activity was increased. The nuclear translocation of TRPV5 increased, and the transcription of the target gene downstream of TRPV5 increased $(\mathrm{P}<0.05)$. In contrast, the expression of overexpressed NHX1 group was significantly increased, the activity of podocytes was decreased, the nuclear access of TRPV5 was decreased, and the transcription of the target gene downstream of TRPV5 was decreased $(\mathrm{P}<0.05)$. NHX1 may reduce podocyte injury in diabetic nephropathy by inhibiting TRPV5 entry into the nucleus.
\end{abstract}

Keywords: Diabetic Nephropathy, Podocyte, NHX1 Protein, TRPV5 Protein

\section{Introduction}

Diabetic nephropathy (DN) is one of the major chronic complications of diabetes and a major cause of end-stage renal disease [1]. Podocyte injury plays a crucial role in the progression of diabetic nephropathy and has received the attention of researchers in recent years [2]. Researches at home and abroad and our research team found that the nuclear transcription factor TRPV5 is a key factor affecting podocyte injury, apoptosis and glomerular sclerosis. In animal models of chronic kidney disease, including lipopolysaccharide nephropathy, 5 / 6 nephrectomy nephropathy and diabetic nephropathy animal model, podocyte TRPV5 plays an important role in pathogenesis [3]. These evidences prompted us to further consider the involvement of podocyte NHX1 in the regulation of the PI3K pathway in peripheral CD4 $+\mathrm{T}$ cells [4].
In the field of kidney disease research, large-scale exome analysis shows that NHX1 plays an important role in the development of the kidney [5]. However, the function of NHX1 in glomerular podocytes has not been reported at home and abroad. This study intends to observe the expression of NHX1 in podocytes through human diabetic nephropathy and cell model, and verify the effect of NHX1 on podocyte injury and activity in vitro, and further explore whether NHX1 can affect podocyte injury by affecting TRPV5 entry influences.

\section{Materials and Methods}

\subsection{Main Instruments and Reagents}

The carbon dioxide incubator was purchased from Thermo; the benchtop centrifuge and sampler were purchased from 
Eppendorf; the frozen high speed centrifuge was purchased from Beckman; the electrophoresis system was purchased from Bio-Rad; and the laser confocal microscope was purchased from Zeiss. Fetal bovine serum, 0.05\% trypsin and Opti-MEM medium were purchased from Gibco; DMEM medium was purchased from Corning; interferon $\gamma$ was purchased from ProSpec; nucleoplasm protein extraction kit was purchased from Guangzhou Kaiji Biotechnology Co., Ltd.; TRPV5 antibody Purchased from Abcam; GAPDH antibody was purchased from Bioworld; Histone antibody and DAPI were purchased from Cell Signaling Technology; anti-podocin antibody, Tris, glycine, acrylamide and type I rat tail collagen were purchased from Sigma; anti-synaptopodin, NHX1 and TRPV5 antibodies were purchased from Santa Cruz; anti-rabbit fluorescence II anti-488 was purchased from Thermo Fisher Scientific; reverse transcription kit and SYBRGREEN were purchased from TaKaRa; goat anti-mouse fluorescent II anti-546, Lipofectamine 2000 and TRIzol were purchased from Life Technologies; NHX1 siRNA was purchased from Guangzhou Ruibo Biotech Co., Ltd..

\subsection{Podocyte Culture and Experimental Grouping Conditions Immortalized Mice}

The podocytes were firstly propagated in a $33^{\circ} \mathrm{C}, 5 \% \mathrm{CO}_{2}$ incubator. When the cell fusion reached $70 \%$ to $80 \%$, they were transferred to a $5 \% \quad \mathrm{CO}_{2}, \quad 37^{\circ} \mathrm{C}$ incubator for differentiation, and cultured at $37^{\circ} \mathrm{C}$ for 10 to 12 days. All podocyte experiments in this experiment were performed after differentiation and maturation.

Grouped as follows: Normal control group (control group): DMEM cultured with $5.3 \mathrm{mmol} / \mathrm{L}$ glucose for $48 \mathrm{~h}$; Mannitol control group: DMEM culture containing $5.3 \mathrm{mmol} / \mathrm{L}$ glucose and $24.7 \mathrm{mmol} / \mathrm{L}$ mannitol $48 \mathrm{~h}$; HG group: DMEM containing $30 \mathrm{mmol} / \mathrm{L}$ glucose for $48 \mathrm{~h}$. After virus-infected podocytes differentiated and matured, add $5 \mu \mathrm{L}$ of adenovirus solution to $5 \mathrm{~mL}$ serum-free DMEM medium. After 24 hours of infection, the culture medium in the culture dish was aspirated and replaced with DMEM containing 5\% serum. Culture medium and other interventions.

\section{3. $\operatorname{SiRNA}$}

After the transfected podocytes were differentiated and matured, a mixture of $50 \mathrm{nmol} / \mathrm{L}$ siRNA and $8 \mu \mathrm{L}$ Lipofectamine 2000 was added to the serum-free antibiotic-free culture medium. After 6 hours, the cells were replaced with 5\% serum DMEM medium for 48 hours. Immunofluorescence staining of renal tissue or podocytes was first performed with ice-methanol fixation, $0.5 \%$ Triton X-100 permeabilization, 5\% BSA blocking, and then incubation at $4^{\circ} \mathrm{C}$ overnight, then incubated with the corresponding fluorescent II anti-light and DAPI The nuclei, the seals, the laser confocal microscope or the fluorescence microscope were used, and the pictures used were taken under a 400 -fold microscope.

\subsection{Western Blot}

After collecting the protein, the protein concentration was measured by BCA method, and the protein denaturant was added to boil and denature. The protein was added to $7.5 \%, 9 \%$ gel for SDS-PAGE, $200 \mathrm{~mA}$ constant flow wet to PVDF membrane, $5 \%$ milk was blocked for $1 \mathrm{~h}$, I was incubated at $4{ }^{\circ} \mathrm{C}$ overnight, and incubated with the corresponding II anti-room temperature for $1 \mathrm{~h}$. Thereafter, exposure was performed using an electrochemiluminescence solution (ECL). The ImageJ software analyzes the grayscale values of the strips. Each set of experiments was repeated 3 times. Total RNA was extracted by RT-PCR TRIzol method, and RNA was reverse transcribed into cDNA using a reverse transcription kit. The expression of the target gene was detected according to the instructions of the SYBR Green PCR kit.

\subsection{Scratch Test}

The well-differentiated podocytes were seeded into 6-well plates at a dose of $1 \times 108 / \mathrm{L}$ per well, and incubated overnight at $5 \% \mathrm{CO}_{2}$ and $37^{\circ} \mathrm{C}$. The cells on each coverslip were scraped off with a $1 \mathrm{~mL}$ sterile tip, washed once with PBS, and replaced with fresh medium for overexpression or silencing. After the above experimental time, high glucose treatment was performed. $\mathrm{h}$. After the treatment, the supernatant was removed and washed twice with PBS, fixed in ice-methanol at $-20^{\circ} \mathrm{C}$ for $15 \mathrm{~min}$, washed three times with PBS, $0.5 \%$ Triton X-100 permeabilized for $10 \mathrm{~min}$, and washed three times with PBS. DAPI staining for $10 \mathrm{~min}$ showed the nuclei, washed 3 times in dark PBS, and mounted under a fluorescence microscope. The images used were taken at 100 magnification.

\subsection{Statistical Processing}

The normal distribution measurement data were expressed as mean \pm standard deviation (mean \pm SD), and one-way analysis of variance was used for comparison between groups. The LSD test was used to compare the two groups. Statistical analysis was performed using statistical software SPSS 22.0, with a statistical significance of $\mathrm{P}<0.05$.

\section{Results}

\subsection{NHX1 Expression Is Reduced in Podocytes of Patients with Diabetic Nephropathy}

Immunofluorescence staining was used to localize podocytes by the glomerular podocyte marker protein synaptopodin. The results showed that the expression of NHX1 was higher in podocytes of normal kidney tissues, while the expression of NHX1 was significantly decreased in glomerular podocytes of patients with diabetic nephropathy., see figure 1 . 


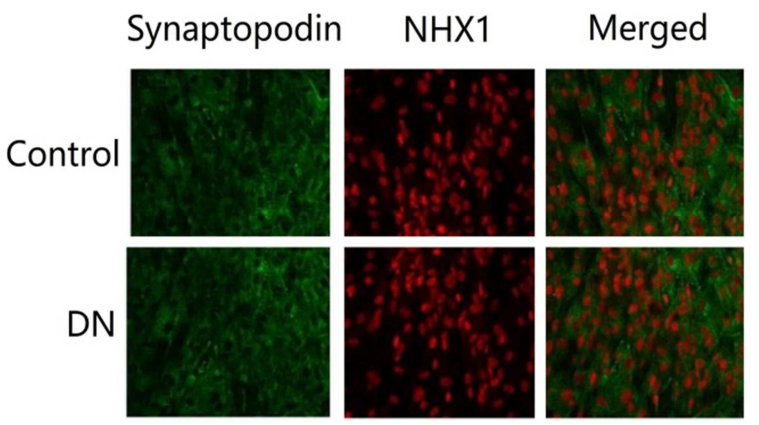

Figure 1. NHX1 expression is reduced in podocytes of patients with diabetic nephropathy.

\subsection{NHX1 Expression Is Decreased in Podocytes Stimulated by High Glucose in Vitro}

The podocytes were treated with $30 \mathrm{mmol} / \mathrm{L}$ glucose for 48 h. RT-PCR and Western blot showed that the expression of NHX1 in the high glucose group was significantly lower than that in the control group and the mannitol group $(\mathrm{P}<0.05)$. After fluorescent staining, DAPI was used to localize the nucleus, and the glomerular podocyte marker protein synaptopodin was used to localize podocytes. The superimposed results showed that $\mathrm{HG}$ intervention in podocytes could induce NHX1 expression significantly reduced. See figure 2.
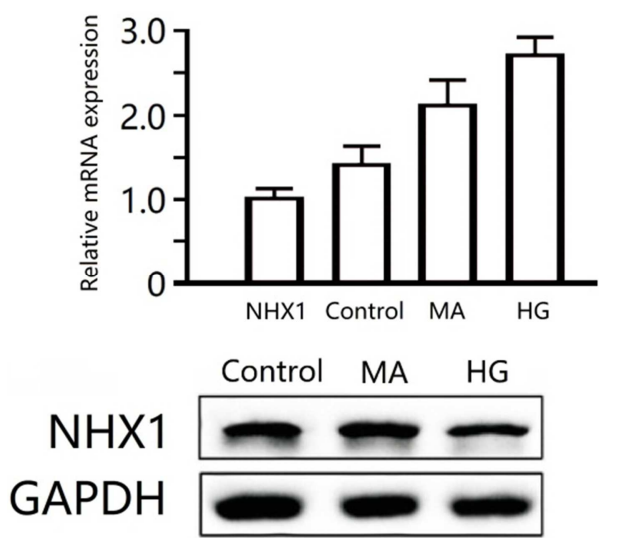

Figure 2. NHXI expression is decreased in podocytes stimulated by high glucose in vitro.

\subsection{Verification of NHX1 Efficiency in Overexpressed and Silenced Podocytes}

RT-PCR results showed that the silence level of NHX1 siRNA1 reached $46 \%$ of the control group $(\mathrm{P}<0.05)$, and the silence level of NHX1 siRNA2 reached $46 \%$ of the control group $(\mathrm{P}<0.05)$. The overexpression level of NHX1 was $\mathrm{OK}$. Reached 15.98 times of the control group $(\mathrm{P}<0.05)$. At the same time, the results of Western blot showed that the silence level of NHX1 siRNA1 reached $36 \%$ of the control group $(\mathrm{P}<$ $0.05)$, and the silence level of NHX1 siRNA2 reached $44 \%$ of the control group $(\mathrm{P}<0.05)$, and the overexpression level of NHX1 It can reach 5.67 times of the control group $(\mathrm{P}<0.05)$. Western blot analysis showed that the expression of podocin was significantly decreased after silencing NHX1 $(\mathrm{P}<0.05)$, and the expression of podocon was significantly increased after overexpression of NHX1 under high glucose stimulation $(\mathrm{P}<0.05)$. Immunofluorescence results showed that after silencing NHX1, the expression of podocin was significantly damaged and disordered compared with the control group, as shown in figure 3.

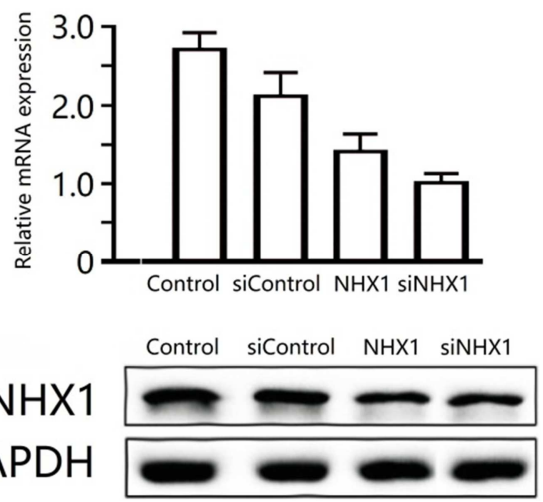

Figure 3. Verification of NHX1 efficiency in overexpressed and silenced podocytes.

\subsection{Regulate the Effect of NHX1 on Podocyte Activity}

The activity of podocytes under different conditions was observed by scratch test. The results showed that when NHX1 was silenced, the number of podocyte migration was significantly increased compared with control group and control siRNA group $(\mathrm{P}<0.05)$. Conversely, under high glucose conditions, when overexpressing NHX1, the number of podocyte migration was significantly reduced compared to the pHBAd + HG group $(\mathrm{P}<0.05)$, see figure 4 .

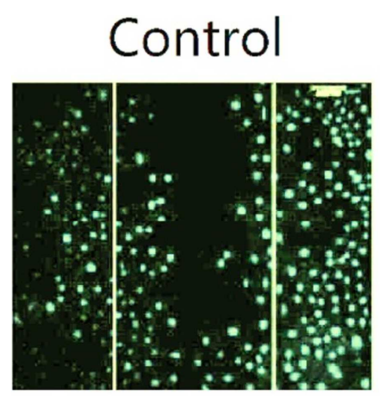

siControl
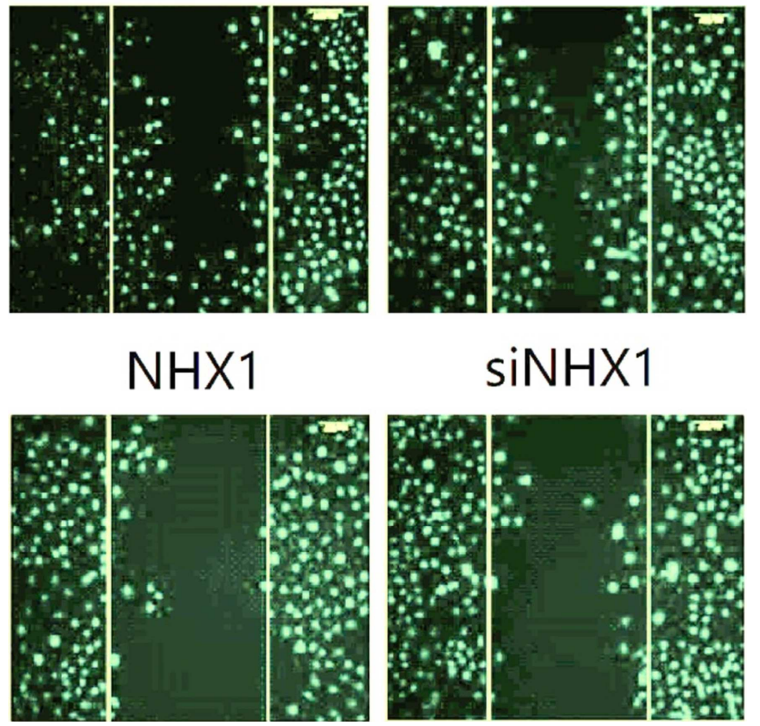

$\operatorname{siNHX1}$

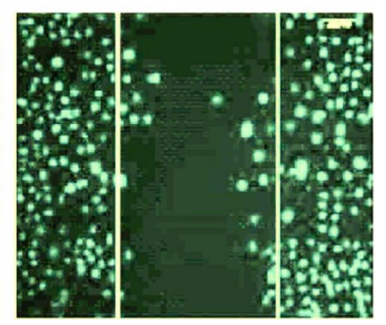

Figure 4. Regulate the effect of $N H X 1$ on podocyte activity.

\section{Discussion}

Diabetic nephropathy is one of the global health problems, and it is the kidney damage caused by diabetes. Its pathogenesis 
is closely related to many factors [6]. Podocytes are an important component of the glomerular filtration barrier, and almost all glomerular diseases are associated with podocyte injury [7]. Therefore, elucidating the mechanism of podocyte injury in diabetic nephropathy will help provide a new strategy for the prevention and treatment of DN. Previous studies have shown that [8], NHX1 can convert the phosphate group into GTP in GDP, which activates Ras. NHX1 protein is expressed in the glomeruli and renal tubules of human kidneys [9]. In 2016, a large-scale exome analysis showed that NHX1 regulates kidney development and function [10]. However, in podocytes, the expression and effects of NHX1 have not been reported. This study found for the first time that both human glomerular podocytes and in vitro cultured podocytes have NHX1 expression, and the expression in podocytes of diabetic nephropathy patients is reduced, and the podocytes cultured in vitro are also reduced in high glucose environment, so we It is speculated that changes in the expression of NHX1 are highly likely to be associated with podocyte injury. Studies have confirmed [11] that the pathological manifestations of the podocin-encoding gene NPHS2 knockout mice are severe mesangial sclerosis. Electron microscopy can observe extensive fusion of the foot processes and disappearance of the ruptured membrane. When podocytes are damaged, podocyte marker protein podocin expression is decreased [12]. At the same time, the enhancement of podocyte activity is also an important injury factor for podocytes. In vivo, it can cause the fusion of podocyte foot processes and even podocyte loss leading to proteinuria. In vitro, it shows the enhancement of podocyte activity and the fusion of foot processes. A similar mechanism [13], inhibition of the activity of injured podocytes is recognized as one of the keys to protect podocytes.

In this experiment, in order to explore the effect of NHX1 in podocytes, we first regulated the expression of NHX1 in podocytes in vitro, down-regulated the expression of NHX1 by NHX1 siRNA, and up-regulated the expression of NHX1 by overexpressing the adenovirus expressing NHX1. The damage of podocytes was confirmed by observing the expression of podocin under different conditions [14]. At the same time, the activity of podocytes under different conditions was observed by scratch test. The above results indicate that NHX1 has a protective effect on podocytes, inhibits podocyte migration, and maintains a relatively stable state of podocytes, ie, NHX1 is a protective factor for podocytes [15].

\section{Conclusion}

This study explored the regulatory mechanism of TRPV5 from a new perspective, enriched the CaN-TRPV5 signaling axis mechanism, and provided a new intervention strategy for clinical treatment of diabetic nephropathy. As a guanylate exchange factor, NHX1 regulates the expression of podocin and cell activity in podocytes, and regulates the entry of TRPV5 into the nucleus; and the immunofluorescence staining of human diabetic kidney tissue is observed. The expression of NHX1 is decreased in cells, and whether NHX1 is also expressed in endothelial cells and mesangial cells. We will continue to explore related problems and construct a transgenic animal model to further verify the effect of NHX1 on renal injury and the relationship with TRPV5. Provide more experimental basis for the application of NHX1 in clinical practice.

\section{References}

[1] Zhou Z, Wan J, Hou X, Geng J, Li X, Bai X. Correction: MicroRNA-27a promotes podocyte injury via PPARgamma-mediated beta-catenin activation in diabetic nephropathy. Cell death \& disease. 2018; 9 (6): 652.

[2] Chen X, Zhao L, Xing Y, Lin B. Down-regulation of microRNA-21 reduces inflammation and podocyte apoptosis in diabetic nephropathy by relieving the repression of TIMP3 expression. Biomedicine \& pharmacotherapy $=$ Biomedecine \& pharmacotherapie. 2018; 108: 7-14.

[3] Zhong F, Chen H, Xie Y, Azeloglu EU, Wei C, Zhang W, et al. Protein S Protects against Podocyte Injury in Diabetic Nephropathy. Journal of the American Society of Nephrology: JASN. 2018; 29 (5): 1397-410.

[4] Bai X, Geng J, Li X, Wan J, Liu J, Zhou Z, et al. Long Noncoding RNA LINC01619 Regulates MicroRNA-27a/Forkhead Box Protein O1 and Endoplasmic Reticulum Stress-Mediated Podocyte Injury in Diabetic Nephropathy. Antioxidants \& redox signaling. 2018; 29 (4): 355-76.

[5] Fujita Y, Tominaga T, Abe H, Kangawa Y, Fukushima N, Ueda $\mathrm{O}$, et al. An adjustment in BMP4 function represents a treatment for diabetic nephropathy and podocyte injury. Scientific reports. 2018; 8 (1): 13011.

[6] Zhang Z, Li X, Liu L, Sun J, Wang X, Zhao Z, et al. Tiaolipiwei Acupuncture Reduces Albuminuria by Alleviating Podocyte Lesions in a Rat Model of Diabetic Nephropathy. Evidence-based complementary and alternative medicine: eCAM. 2018; 2018: 1913691.

[7] He Y, Zhang M, Wu Y, Jiang H, Fu H, Cai Y, et al. Aberrant activation of Notch-1 signaling inhibits podocyte restoration after islet transplantation in a rat model of diabetic nephropathy. Cell death \& disease. 2018; 9 (10): 950.

[8] Zhang Y, Chen X, Yuan L, Wu J, Guo N, Liu J. Down-regulation of IRAK1 attenuates podocyte apoptosis in diabetic nephropathy through PI3K/Akt signaling pathway. Biochemical and biophysical research communications. 2018; 506 (3): 529-35.

[9] Lei X, Zhang L, Li Z, Ren J. Astragaloside IV/lncRNA-TUG1/TRAF5 signaling pathway participates in podocyte apoptosis of diabetic nephropathy rats. Drug design, development and therapy. 2018; 12: 2785-93.

[10] Wang Y, Li H, Song SP. beta-Arrestin 1/2 Aggravates Podocyte Apoptosis of Diabetic Nephropathy via Wnt/beta-Catenin Pathway. Medical science monitor: international medical journal of experimental and clinical research. 2018; 24: 1724-32.

[11] Pan Y, Jiang S, Hou Q, Qiu D, Shi J, Wang L, et al. Dissection of Glomerular Transcriptional Profile in Patients With Diabetic Nephropathy: SRGAP2a Protects Podocyte Structure and Function. Diabetes. 2018; 67 (4): 717-30. 
[12] Wang XB, Zhu H, Song W, Su JH. Gremlin Regulates Podocyte Apoptosis via Transforming Growth Factor-beta (TGF-beta) Pathway in Diabetic Nephropathy. Medical science monitor: international medical journal of experimental and clinical research. 2018; 24: 183-9.

[13] Lv Z, Hu M, Fan M, Li X, Lin J, Zhen J, et al. Podocyte-specific Rac1 deficiency ameliorates podocyte damage and proteinuria in STZ-induced diabetic nephropathy in mice. Cell death \& disease. 2018; 9 (3): 342.
[14] Wang X, Gao L, Lin H, Song J, Wang J, Yin Y, et al. Mangiferin prevents diabetic nephropathy progression and protects podocyte function via autophagy in diabetic rat glomeruli. European journal of pharmacology. 2018; 824: 170-8.

[15] Tung CW, Hsu YC, Shih YH, Chang PJ, Lin CL. Glomerular mesangial cell and podocyte injuries in diabetic nephropathy. Nephrology (Carlton). 2018; 23 Suppl 4: 32-7. 